\title{
OPTIMAL EXTINCTION RATES FOR THE FAST DIFFUSION EQUATION WITH STRONG ABSORPTION
}

\author{
RAZVAN GABRIEL IAGAR AND PHILIPPE LAURENÇOT
}

\begin{abstract}
Optimal extinction rates near the extinction time are derived for non-negative solutions to a fast diffusion equation with strong absorption, the power of the absorption exceeding that of the diffusion.
\end{abstract}

\section{INTRODUCTION}

Given $m \in(0, \infty), q \in(0,1)$, and a non-negative initial condition $u_{0}$ in $B C\left(\mathbb{R}^{N}\right), u_{0} \not \equiv 0$, it is well-known that the initial value problem

$$
\begin{aligned}
\partial_{t} u-\Delta u^{m}+u^{q} & =0, \quad(t, x) \in(0, \infty) \times \mathbb{R}^{N}, \\
u(0) & =u_{0}, \quad x \in \mathbb{R}^{N},
\end{aligned}
$$

has a unique non-negative (weak) solution $u$ which vanishes identically after a finite time, a phenomenon usually referred to as finite time extinction [19]21. More precisely, introducing the extinction time

$$
T_{e}:=\sup \{t>0: u(t) \not \equiv 0\}>0,
$$

then $T_{e}$ is finite and satisfies $T_{e} \leq\left\|u_{0}\right\|_{\infty}^{(1-q)} /(1-q)$, the latter upper bound being a straightforward consequence of (1.1) and the comparison principle. Moreover, there holds

$$
u(t) \not \equiv 0 \text { for } t \in\left[0, T_{e}\right) \text { and } u(t) \equiv 0 \text { for } t \geq T_{e} .
$$

When $q<m$ and $u_{0}(x) \rightarrow 0$ as $|x| \rightarrow \infty$, finite time extinction is accompanied by an even more striking phenomenon, the instantaneous shrinking of the support, that is, the positivity set $\mathcal{P}(t):=$ $\left\{x \in \mathbb{R}^{N}: u(t, x)>0\right\}$ of $u$ at time $t$ is a relatively compact subset of $\mathbb{R}^{N}$ for all $t \in\left(0, T_{e}\right)$, even if $\mathcal{P}(0)=\mathbb{R}^{N}$ initially [1, 5, 7, 19]. Observe that the inequality $q<m$ is always satisfied when the diffusion is linear $(m=1)$ or slow $(m>1)$. Additional information on the behaviour of $\mathcal{P}(t)$ as $t \rightarrow T_{e}$ is also available when $m \geq 1$ and $N=1$ [6, 10,14].

Once finite time extinction is known to take place, gaining further insight into the underlying mechanism requires to identify the behaviour of $u(t)$ as $t \rightarrow T_{e}$, a preliminary step being to determine

Date: October 14, 2018.

1991 Mathematics Subject Classification. 35B40 - 35K67 - 35K57.

Key words and phrases. Extinction, optimal rates, fast diffusion, strong absorption. 
the relevant space and time scales. Simple scaling arguments predict that, for $r \in[1, \infty]$ and $u_{0} \in L^{r}\left(\mathbb{R}^{N}\right)$, there is a constant $\gamma_{r}>0$ (depending on $N, m, q, u_{0}$, and $r$ ) such that

$$
\|u(t)\|_{r} \sim \gamma_{r}\left(T_{e}-t\right)^{\alpha-(N \beta / r)}
$$

where

$$
\alpha:=\frac{1}{1-q}>0, \quad \beta:=\frac{q-m}{2(1-q)} \in \mathbb{R} .
$$

As already observed by several authors [9, 12, 15, a rather simple comparison argument provides a lower bound for the $L^{\infty}$-norm of the form (1.4). Indeed, consider $t \in\left(0, T_{e}\right)$ and let $x(t) \in \mathbb{R}^{N}$ be a point where $u(t)$ reaches its maximum value, that is, $u(t, x(t))=\|u(t)\|_{\infty}$. Then $u(t)^{m}$ also attains its maximum value at this point, so that $\Delta u^{m}(t, x(t)) \leq 0$ and we infer from (1.1a) that (at least formally)

$$
\frac{d}{d t}\|u(t)\|_{\infty}=\partial_{t} u(t, x(t)) \leq-u(t, x(t))^{q}=-\|u(t)\|_{\infty}^{q} .
$$

Integrating the above differential inequality over $\left(t, T_{e}\right)$ gives the expected lower bound

$$
\|u(t)\|_{\infty} \geq\left[(1-q)\left(T_{e}-t\right)\right]^{1 /(1-q)}, \quad t \in\left[0, T_{e}\right)
$$

The derivation of an upper bound of the form (1.4) turns out to be more involved and the results obtained so far are rather sparse: in one space dimension, the upper bound

$$
\|u(t)\|_{\infty} \leq C_{\infty}\left(T_{e}-t\right)^{1 /(1-q)}, \quad t \in\left[0, T_{e}\right)
$$

is shown in [17, Proposition 2.2] for $m=1$ and in [9, Lemma 5.2, Lemma 7.2 \& Lemma 9.2] for $m \in(0,1)$, the latter being valid only for compactly supported initial data. The proofs are however of a completely different nature: in [17, properties of the linear heat equation are used while the approach in [9] relies on the intersection-comparison technique, which requires in particular the compactness of the support of the initial condition. Still for $m=1$ but in any space dimension, the upper bound (1.7) is derived in [12, Lemma 2.1] for radially symmetric initial data $u_{0}$ having a non-increasing profile and satisfying $\Delta u_{0}+\mu u_{0}^{q} \geq 0$ in $\mathbb{R}^{N}$ for some $\mu>0$. The last case for which (1.7) is proved corresponds to the choice $m=2-q>1$ and the proof relies on the derivation of an Aronson-Bénilan estimate, which seems to be only available for this specific choice of the parameters $m$ and $q$ [15].

The purpose of this note is to contribute to the validity of (1.4) and derive optimal upper and lower bounds near the extinction time when the parameters $m$ and $q$ range in

$$
\frac{(N-2)_{+}}{N}<m<q<1 .
$$

Recalling that a lower bound in $L^{\infty}$ is already available, see (1.6), we begin with upper bounds.

Theorem 1.1 (Upper bounds). Assume that $m$ and $q$ satisfy (1.8) and consider a non-negative initial condition $u_{0} \in B C\left(\mathbb{R}^{N}\right), u_{0} \not \equiv 0$, for which there is $\kappa_{0}>0$ such that

$$
u_{0}(x) \leq \kappa_{0}|x|^{-2 /(q-m)}, \quad x \in \mathbb{R}^{N} .
$$


Given $r \in[1, \infty]$, there is $C_{r}>0$ depending only on $N, m, q, u_{0}$, and $r$ such that the solution $u$ to (1.1a) $-(1.1 \mathrm{~b})$ satisfies

$$
\|u(t)\|_{r} \leq C_{r}\left(T_{e}-t\right)^{\alpha-(N \beta / r)}, \quad t \in\left(0, T_{e}\right)
$$

the extinction time $T_{e}$ being defined in (1.2).

Theorem 1.1 thus extends the validity of the upper bound (1.7) established in [9] for $N=1$ and $r=\infty$ to any space dimension $N \geq 1$ and $r \in[1, \infty]$, while relaxing the assumption of compact support required in [9]. It is worth mentioning that the validity of (1.10) for $r \in[1, \infty)$ does not seem to be a simple consequence of (1.10) for $r=\infty$ since $u(t)$ is positive everywhere in $\mathbb{R}^{N}$ for all $t \in\left(0, T_{e}\right)$ even if $u_{0}$ is compactly supported, see [9, Lemma 2.5] and Proposition 1.4 below.

To be able to cope with higher space dimensions and non-compactly supported initial data, the proof of Theorem 1.1 takes a different route from that in [9] and is carried out in two steps: we first show that the algebraic decay at infinity (1.9) enjoyed by $u_{0}$ remains true throughout time evolution and combine it with (1.1a) to prove (1.10) for $r=1$. We next use self-similar variables and Moser's interation technique to derive (1.10) for all $r \in(1, \infty]$.

As a consequence of (1.6) and Theorem 1.1 for $r=\infty$, the correct time scale for the extinction phenomenon is identified. We now supplement the lower bound (1.6) in $L^{\infty}$ with another one in $L^{m+1}$. On the one hand, it allows us to identify the right space scale. On the other hand, its derivation does not rely on the comparison principle but on energy estimates, a technique which is more likely to extend to other problems for which the former might not be available.

Theorem 1.2 (Lower bound in $\left.L^{m+1}\right)$. Assume that $m$ and $q$ satisfy (1.8) and consider a nonnegative initial condition $u_{0} \in B C\left(\mathbb{R}^{N}\right), u_{0} \not \equiv 0$, such that $u_{0} \in L^{m+1}\left(\mathbb{R}^{N}\right)$. There is $c_{m+1}>0$ depending only on $N, m, q$, and $u_{0}$ such that the solution $u$ to (1.1a $)-(1.1 \mathrm{~b})$ satisfies

$$
\|u(t)\|_{m+1} \geq c_{m+1}\left(T_{e}-t\right)^{\alpha-(N \beta /(m+1))}, \quad t \in\left(0, T_{e}\right)
$$

the extinction time $T_{e}$ being defined in (1.2).

Observing that Theorems 1.1 and 1.2 are shown without using the $L^{\infty}$-lower bound (1.6), the latter may be recovered from these two results by Hölder's inequality, with a less explicit constant though.

Corollary 1.3. Assume that $m$ and $q$ satisfy (1.8) and consider a non-negative initial condition $u_{0} \in B C\left(\mathbb{R}^{N}\right), u_{0} \neq \equiv 0$, enjoying the decay property (1.9). For $r \in(m+1, \infty]$, there is $c_{r}>0$ depending only $N, m, q, u_{0}$, and $r$ such that the solution $u$ to (1.1a)-(1.1b) satisfies

$$
\|u(t)\|_{r} \geq c_{r}\left(T_{e}-t\right)^{\alpha-(N \beta / r)}, \quad t \in\left(0, T_{e}\right) .
$$

Summarizing the outcome of Theorem 1.1, Theorem 1.2, and Corollary 1.3, we have shown that, for all non-negative initial data $u_{0} \in B C\left(\mathbb{R}^{N}\right), u_{0} \not \equiv 0$, enjoying the decay property (1.9), the corresponding solution $u$ to (1.1a $)$ - (1.1b) is bounded in $L^{r}\left(\mathbb{R}^{N}\right), r \in[m+1, \infty]$, from above and from below at time $t \in\left(0, T_{e}\right)$ by the same power of $T_{e}-t$. Such estimates pave the way towards a more precise description of the behaviour of $u(t)$ as $t \rightarrow T_{e}$, which is expected to be self-similar. That this 
is indeed the case is shown in [8,9] in one space dimension, another building block of the proof being the uniqueness of self-similar solutions [8].

We end up this note with the already mentioned everywhere positivity of solutions to (1.1a) $-(1.1 \mathrm{~b})$ for positive times prior to the extinction time. As we shall see below, this property holds true for a wider range of the parameters $m$ and $q$, namely $0<m \leq q<1$. It is already observed in [9, Lemma 2.5] in one space dimension and we extend it herein to any space dimension. It is worth emphasizing that it includes the case $q=m$ and contrasts markedly with the instantaneous shrinking of the support occurring when $q<m$.

Proposition 1.4 (Everywhere positivity). Consider $0<m \leq q<1$. Let $u_{0} \in B C\left(\mathbb{R}^{N}\right)$ be a nonnegative initial condition, $u_{0} \not \equiv 0$, and denote the corresponding solution to (1.1a) $-(1.1 \mathrm{~b})$ by $u$ with extinction time $T_{e}$. For $t \in\left(0, T_{e}\right)$, there holds

$$
\mathcal{P}(t):=\left\{x \in \mathbb{R}^{N}: u(t, x)>0\right\}=\mathbb{R}^{N} .
$$

Before proving the results stated above, we point out once more that the energy techniques developed herein seem to be rather flexible and are expected to have a wider range of applicability. For instance, a related approach is used in the companion paper [18], where optimal (lower and upper) bounds near the extinction time are established for a different fast diffusion equation (featuring the $p$-Laplacian operator, $p \in(1,2))$ with a gradient absorption term.

\section{UPPER BOUNDS NEAR THE EXTINCTION TIME}

Throughout this section, we assume that $m$ and $q$ satisfy (1.8) and consider a non-negative initial condition $u_{0} \in B C\left(\mathbb{R}^{N}\right), u_{0} \not \equiv 0$, enjoying the decay property (1.9). Let $u$ be the corresponding solution to (1.1a $)-(1.1 \mathrm{~b})$.

2.1. $L^{1}$-estimate. We begin with the propagation throughout time evolution of the algebraic decay (1.9) and set

$$
\kappa_{*}:=\left(\frac{2 m(m+q)}{(q-m)^{2}}\right)^{1 /(q-m)} .
$$

Lemma 2.1. For $t \in[0, \infty)$ and $x \in \mathbb{R}^{N} \backslash\{0\}$, there holds

$$
u(t, x) \leq \max \left\{\kappa_{0}, \kappa_{*}\right\}|x|^{-2 /(q-m)} .
$$

Proof. Set $\Sigma_{\kappa}(x):=\kappa|x|^{-2 /(q-m)}$ for $x \in \mathbb{R}^{N} \backslash\{0\}$, where $\kappa$ is a positive constant yet to be determined. We note that

$$
\begin{aligned}
-\Delta \Sigma^{m}(x)+\Sigma(x)^{q}= & -\kappa^{m}\left[\frac{2 m(m+q)}{(q-m)^{2}}|x|^{-2 q /(q-m)}-\frac{2 m(N-1)}{(q-m)}|x|^{-2 q /(q-m)}\right] \\
& +\kappa^{q}|x|^{-2 q /(q-m)} \\
\geq & \kappa^{m}\left(\kappa^{q-m}-\kappa_{*}^{q-m}\right)|x|^{-2 q /(q-m)}
\end{aligned}
$$

for $x \in \mathbb{R}^{N} \backslash\{0\}$, so that $\Sigma_{\kappa}$ is a supersolution to (1.1a) in $\mathbb{R}^{N} \backslash\{0\}$ for all $\kappa \geq \kappa_{*}$. We then choose $\kappa=\max \left\{\kappa_{0}, \kappa_{*}\right\}$ and use the comparison principle to complete the proof of Lemma 2.1. 
We are now in a position to derive the claimed upper bound near the extinction time for $r=1$. Proof of Theorem 1.1: $r=1$. Let $t \in\left[0, T_{e}\right)$. Integrating (1.1a) over $\left(t, T_{e}\right) \times \mathbb{R}^{N}$ gives

$$
\|u(t)\|_{1}=\int_{t}^{T_{e}} \int_{\mathbb{R}^{N}} u(s, x)^{q} d x d s .
$$

Owing to (1.8), there holds $2 q /(q-m)>N$ and we infer from Lemma 2.1 and Hölder's inequality that, for $s \in\left(t, T_{e}\right)$ and $R>0$,

$$
\begin{aligned}
\int_{\mathbb{R}^{N}} u(s, x)^{q} d x & =\int_{B(0, R)} u(s, x)^{q} d x+\int_{\mathbb{R}^{N} \backslash B(0, R)} u(s, x)^{q} d x \\
& \leq C R^{N(1-q)}\|u(s)\|_{1}^{q}+\left(\max \left\{\kappa_{0}, \kappa_{*}\right\}\right)^{q}\left|\mathbb{S}^{N-1}\right| \int_{R}^{\infty} r^{N-1-(2 q /(q-m))} d r \\
& \leq C\left(R^{N(1-q)}\|u(s)\|_{1}^{q}+R^{(N(q-m)-2 q) /(q-m)}\right) .
\end{aligned}
$$

We next optimize in $R$ in the previous inequality by setting $R(s):=\|u(s)\|_{1}^{-(q-m) /(N(m-q)+2)}$, which satisfies

$$
R(s)^{N(1-q)}\|u(s)\|_{1}^{q}=R(s)^{(N(q-m)-2 q) /(q-m)}=\|u(s)\|_{1}^{(N(m-q)+2 q) /(N(m-q)+2)} .
$$

Consequently, taking $R=R(s)$ in the previous inequality, we obtain

$$
\int_{\mathbb{R}^{N}} u(s, x)^{q} d x \leq C\|u(s)\|_{1}^{(N(m-q)+2 q) /(N(m-q)+2)},
$$

which gives, together with (2.2), the positivity of $N(m-q)+2 q$, and the time monotonicity of $s \mapsto\|u(s)\|_{1}$,

$$
\begin{aligned}
\|u(t)\|_{1} & \leq C \int_{t}^{T_{e}}\|u(s)\|_{1}^{(N(m-q)+2 q) /(N(m-q)+2)} d s \\
& \leq C\left(T_{e}-t\right)\|u(t)\|_{1}^{(N(m-q)+2 q) /(N(m-q)+2)},
\end{aligned}
$$

from which (1.10) for $r=1$ readily follows.

2.2. Scaling variables and $L^{r}$-estimates, $r \in(1, \infty]$. The next step is to take advantage of the just derived $L^{1}$-upper bound to derive the corresponding ones in $L^{r}$ for $r \in(1, \infty]$. To this end, we introduce the scaling variables

$$
s:=\ln \left(\frac{T_{e}}{T_{e}-t}\right), \quad y:=x\left(T_{e}-t\right)^{\beta}, \quad(t, x) \in\left[0, T_{e}\right) \times \mathbb{R}^{N},
$$

and the new unknown function $v$ defined by

$$
u(t, x)=\left(T_{e}-t\right)^{\alpha} v\left(\ln \left(T_{e}\right)-\ln \left(T_{e}-t\right), x\left(T_{e}-t\right)^{\beta}\right), \quad(t, x) \in\left[0, T_{e}\right) \times \mathbb{R}^{N},
$$

or, equivalently,

$$
v(s, y)=T_{e}^{-\alpha} e^{\alpha s} u\left(T_{e}\left(1-e^{-s}\right), y T_{e}^{-\beta} e^{\beta s}\right), \quad(s, y) \in[0, \infty) \times \mathbb{R}^{N} .
$$


It readily follows from (1.1a) $-(1.1 \mathrm{~b})$ that $v$ solves

$$
\begin{aligned}
\partial_{s} v(s, y) & =\alpha v(s, y)+\beta y \cdot \nabla v(s, y)+\Delta v^{m}(s, y)-v(s, y)^{q}, \quad(s, y) \in(0, \infty) \times \mathbb{R}^{N}, \\
v(0, y) & =v_{0}(y):=T_{e}^{-\alpha} u_{0}\left(y T_{e}^{-\beta}\right), \quad y \in \mathbb{R}^{N} .
\end{aligned}
$$

Since

$$
\|u(t)\|_{r}=\left(T_{e}-t\right)^{\alpha-(N \beta / r)}\|v(s)\|_{r}, \quad t \in\left(0, T_{e}\right)
$$

for all $r \in[1, \infty]$, we realize that an upper bound such as (1.10) on $\|u(t)\|_{r}$ for $t \in\left(0, T_{e}\right)$ obviously follows from a uniform upper bound on $\|v(s)\|_{r}$ for $s \geq 0$, the converse being true as well. In particular, it follows from (2.8) and Theorem 1.1 for $r=1$ that

$$
\|v(s)\|_{1} \leq C_{1}, \quad s \geq 0
$$

and we may assume without loss of generality that $C_{1} \geq 1$.

We now aim at using a bootstrap argument to deduce from (2.6) and (2.9) that $v$ belongs to $L^{\infty}\left(0, \infty ; L^{r}\left(\mathbb{R}^{N}\right)\right)$ for all $r \in(1, \infty]$. To this end, Moser's iteration technique is a suitable tool and the way we apply it is inspired from [2, Theorem 3.1]. But since [2, Theorem 3.1] is devoted to the slow diffusion case $m>1$, some technical aspects of its proof do not seem to apply directly here and we borrow additional arguments from the proof of [3, Proposition 2].

Lemma 2.2. Let $r \in(0, \infty]$. There is $C_{r+1}>0$ depending only on $N, m, q, u_{0}$, and $r$ such that

$$
\|v(s)\|_{r+1} \leq C_{r+1}, \quad s \geq 0
$$

Proof. Let $r \in[2-m, \infty)$. Multiplying (2.6) by $v^{r}$, integrating over $\mathbb{R}^{N}$, and using integration by parts, we obtain

$$
\begin{gathered}
\frac{1}{r+1} \frac{d}{d s}\|v\|_{r+1}^{r+1}+r m \int_{\mathbb{R}^{N}} v^{r+m-2}|\nabla v|^{2} d y+\int_{\mathbb{R}^{N}} v^{r+q} d y=\left(\alpha-\frac{N \beta}{r+1}\right)\|v\|_{r+1}^{r+1}, \\
\frac{d}{d s}\|v\|_{r+1}^{r+1}+\frac{4 m r(r+1)}{(m+r)^{2}}\left\|\nabla v^{(m+r) / 2}\right\|_{2}^{2} \leq \alpha(r+1)\|v\|_{r+1}^{r+1} .
\end{gathered}
$$

Since $4 m r(r+1) \geq 2 m(m+r)^{2}$, we end up with

$$
\frac{d}{d s}\|v\|_{r+1}^{r+1}+2 m\left\|\nabla v^{(m+r) / 2}\right\|_{2}^{2} \leq \alpha(r+1)\|v\|_{r+1}^{r+1} .
$$

We next fix $\zeta \in\left(2 / m, 2^{*}\right)$ where $2^{*}:=2 N /(N-2)_{+}$(with $2^{*}=\infty$ for $\left.N=1,2\right)$. On the one hand, it follows from the Gagliardo-Nirenberg inequality that

$$
\left\|v^{(m+r) / 2}\right\|_{\zeta} \leq C\left\|\nabla v^{(m+r) / 2}\right\|_{2}^{\theta}\left\|v^{(m+r) / 2}\right\|_{1}^{1-\theta}
$$

with

$$
\theta:=\frac{2 N(\zeta-1)}{(N+2) \zeta} .
$$

On the other hand, since $(m+r) / 2 \in[1, \zeta(m+r) / 2]$ for $r \geq 2-m$, we infer from Hölder's inequality that

$$
\|v\|_{(m+r) / 2}^{(m+r) / 2} \leq\|v\|_{\zeta(m+r) / 2}^{\zeta(m+r)(m+r-2) / 2[\zeta(m+r)-2]}\|v\|_{1}^{(\zeta-1)(m+r) /[\zeta(m+r)-2]} .
$$


We deduce from (2.11) and (2.12) that

$$
\begin{aligned}
\|v\|_{\zeta(m+r) / 2}^{(m+r) / 2} & =\left\|v^{(m+r) / 2}\right\|_{\zeta} \leq C\left\|\nabla v^{(m+r) / 2}\right\|_{2}^{\theta}\left(\|v\|_{(m+r) / 2}^{(m+r) / 2}\right)^{1-\theta} \\
& \leq C\left\|\nabla v^{(m+r) / 2}\right\|_{2}^{\theta}\left[\|v\|_{\zeta(m+r) / 2}^{\zeta(m+r)(m+r-2) / 2[\zeta(m+r)-2]}\|v\|_{1}^{(\zeta-1)(m+r) /[\zeta(m+r)-2]}\right]^{1-\theta},
\end{aligned}
$$

hence

$$
\|v\|_{\zeta(m+r) / 2}^{\zeta(m+r)[N(m+r)+2-N] / N[\zeta(m+r)-2]} \leq C\left\|\nabla v^{(m+r) / 2}\right\|_{2}^{2}\|v\|_{1}^{[2 N-(N-2) \zeta](m+r) / N[\zeta(m+r)-2]} .
$$

Moreover, since $\zeta>2 / m$ and $m<1$, we have $2 r \leq m[\zeta(m+r)-2]$, hence

$$
\begin{aligned}
\frac{[2 N-(N-2) \zeta](m+r)}{N[\zeta(m+r)-2]} & \leq \frac{[2 N-(N-2) \zeta]}{N} \frac{m(m+r)}{2 r} \\
& \leq \frac{m[2 N-(N-2) \zeta]}{N}
\end{aligned}
$$

so that

$$
\begin{aligned}
\|v\|_{1}^{[2 N-(N-2) \zeta](m+r) / N[\zeta(m+r)-2]} & \leq C_{1}^{[2 N-(N-2) \zeta](m+r) / N[\zeta(m+r)-2]} \\
& \leq C_{1}^{m[2 N-(N-2) \zeta] / N} .
\end{aligned}
$$

Also,

$$
1-\frac{N[\zeta(m+r)-2]}{\zeta[N(m+r)+2-N]}=\frac{2 N-\zeta(N-2)}{\zeta[N(m+r)+2-N]}>0,
$$

and we infer from (2.13), (2.14), and Young's inequality that

$$
\begin{aligned}
\|v\|_{\zeta(m+r) / 2}^{m+r} \leq & \frac{N[\zeta(m+r)-2]}{\zeta[N(m+r)+2-N]}\|v\|_{\zeta(m+r) / 2}^{\zeta(m+r)[N(m+r)+2-N] / N[\zeta(m+r)-2]} \\
& +\frac{2 N-\zeta(N-2)}{\zeta[N(m+r)+2-N]} \\
\leq & C\left\|\nabla v^{(m+r) / 2}\right\|_{2}^{2}+1
\end{aligned}
$$

Therefore, there is $\nu \in(0,1)$ depending only on $N, m, q$, and $u_{0}$ such that

$$
\nu\left(\|v\|_{\zeta(m+r) / 2}^{m+r}-1\right) \leq\left\|\nabla v^{(m+r) / 2}\right\|_{2}^{2} .
$$


Moreover, since $r+1 \in[1, \zeta(m+r) / 2]$, it follows from (2.9) and Hölder's and Young's inequalities that

$$
\begin{aligned}
\|v\|_{r+1}^{r+1} & \leq\|v\|_{\zeta(m+r) / 2}^{\zeta r(r+m) /[\zeta(r+m)-2]}\|v\|_{1}^{[(\zeta-2) r+\zeta m-2] /(\zeta r+\zeta m-2)} \\
& \leq C_{1}^{[(\zeta-2) r+\zeta m-2] /(\zeta r+\zeta m-2)}\|v\|_{\zeta(m+r) / 2}^{\zeta r(r+m) /[\zeta(r+m)-2]} \\
& \leq C_{1}\|v\|_{\zeta(m+r) / 2}^{\zeta r(r+m) /[\zeta(r+m)-2]} \\
& \leq \frac{\zeta m-2}{\zeta(m+r)-2} C_{1}^{[\zeta(m+r)-2] /(\zeta m-2)}+\frac{\zeta r}{\zeta(m+r)-2}\|v\|_{\zeta(m+r) / 2}^{m+r} \\
& \leq C_{1}^{[\zeta(m+r)-2] /(\zeta m-2)}+\|v\|_{\zeta(m+r) / 2}^{m+r}
\end{aligned}
$$

Next, let $\sigma>1$ to be chosen appropriately later on and set

$$
\mathcal{I}_{r}(s):=\int_{\mathbb{R}^{N}} v(s, y)^{\zeta[r+m+\sigma(1-m)] /[\sigma(\zeta-2)+2]} d y, \quad s \geq 0 .
$$

Since $\sigma(\zeta-2)+2 \in[\zeta, \sigma \zeta]$ and

$$
r+1=\frac{\sigma-1}{\sigma}(m+r)+\frac{m+r+\sigma(1-m)}{\sigma},
$$

we deduce from (2.9) and Hölder's and Young's inequalities that, for $\delta>0$,

$$
\begin{aligned}
\|v\|_{r+1}^{r+1} & \leq\|v\|_{\zeta(m+r) / 2}^{(\sigma-1)(m+r) / \sigma} \mathcal{I}_{r}^{[\sigma(\zeta-2)+2] / \sigma \zeta} \\
& \leq \frac{(\sigma-1) \delta}{\sigma}\|v\|_{\zeta(m+r) / 2}^{m+r}+\frac{1}{\sigma \delta^{\sigma-1}} \mathcal{I}_{r}^{[\sigma(\zeta-2)+2] / \zeta} \\
& \leq \delta\|v\|_{\zeta(m+r) / 2}^{m+r}+\frac{1}{\delta^{\sigma-1}} \mathcal{I}_{r}^{[\sigma(\zeta-2)+2] / \zeta} .
\end{aligned}
$$

Combining (2.10), (2.15), and (2.17) leads us to

$$
\begin{aligned}
\frac{d}{d s}\|v\|_{r+1}^{r+1} & +2 m \nu\left(\|v\|_{\zeta(m+r) / 2}^{m+r}-1\right) \leq \frac{d}{d s}\|v\|_{r+1}^{r+1}+2 m\left\|\nabla v^{(m+r) / 2}\right\|_{2}^{2} \\
& \leq \alpha(r+1)\|v\|_{r+1}^{r+1} \\
& \leq \alpha \delta(r+1)\|v\|_{\zeta(m+r) / 2}^{m+r}+\frac{\alpha(r+1)}{\delta^{\sigma-1}} \mathcal{I}_{r}^{[\sigma(\zeta-2)+2] / \zeta} .
\end{aligned}
$$

We then choose $\delta=m \nu / \alpha(r+1)$ in the above inequality to obtain

$$
\frac{d}{d s}\|v\|_{r+1}^{r+1}+m \nu\|v\|_{\zeta(m+r) / 2}^{m+r} \leq 2 m \nu+\frac{\alpha^{\sigma}(r+1)^{\sigma}}{(m \nu)^{\sigma-1}} \mathcal{I}_{r}^{[\sigma(\zeta-2)+2] / \zeta} .
$$

We finally use (2.16) to estimate from below the second term of the left-hand side of the previous inequality and end up with

$$
\frac{d}{d s}\|v\|_{r+1}^{r+1}+m \nu\|v\|_{r+1}^{r+1} \leq 2 m \nu+m \nu C_{1}^{[\zeta(m+r)-2] /(\zeta m-2)}+\frac{\alpha^{\sigma}(r+1)^{\sigma}}{(m \nu)^{\sigma-1}} \mathcal{I}_{r}^{[\sigma(\zeta-2)+2] / \zeta} .
$$


We first choose

$$
\sigma=\frac{\zeta(m+r)-2}{\zeta m-2}>1
$$

in (2.18) and observe that this choice guarantees that

$$
\zeta[m+r+\sigma(1-m)]=\sigma(\zeta-2)+2 .
$$

Consequently, $\mathcal{I}_{r}=\|v\|_{1}$ and we deduce from (2.9) and (2.18) that there is $C(r)>0$ depending on $N, m, q, u_{0}$, and $r$ such that

$$
\frac{d}{d s}\|v\|_{r+1}^{r+1}+m \nu\|v\|_{r+1}^{r+1} \leq C(r)
$$

Integrating the previous differential inequality entails that

$$
\sup _{s \geq 0}\|v(s)\|_{r+1}<\infty .
$$

The validity of (2.19) extends to all $r \in(0,2-m)$ by (2.9) and Hölder's inequality.

To complete the proof of Lemma 2.2 , we are left to check the boundedness of $v$ in $L^{\infty}\left(\mathbb{R}^{N}\right)$. To this end, we take $\sigma=\sigma_{0}:=2(\zeta-1) /(\zeta-2)>1$ in (2.18) and obtain, after integration with respect to time,

$$
\begin{aligned}
\|v(s)\|_{r+1}^{r+1} \leq & \left\|v_{0}\right\|_{r+1}^{r+1} e^{-m \nu s}+2+C_{1}^{[\zeta(m+r)-2] /(\zeta m-2)} \\
& +\left(\frac{\alpha(r+1)}{m \nu}\right)^{\sigma_{0}}\left[\sup _{s_{*} \in[0, s]} \mathcal{I}_{r}\left(s_{*}\right)\right]^{2} \\
\leq & \left\|v_{0}\right\|_{1}\left\|v_{0}\right\|_{\infty}^{r}+2+C_{1}^{[\zeta(m+r)-2] /(\zeta m-2)} \\
& +\left(\frac{\alpha(r+1)}{m \nu}\right)^{\sigma_{0}}\left[\sup _{s_{*} \in[0, s]} \mathcal{I}_{r}\left(s_{*}\right)\right]^{2},
\end{aligned}
$$

and

$$
\mathcal{I}_{r}=\|v\|_{\left[(r+m)+\sigma_{0}(1-m)\right] / 2}^{\left[(r+m)+\sigma_{0}(1-m)\right] / 2} .
$$

Therefore, there are $K_{0}>0$ and $K_{1}>0$ depending only on $N, m, q$, and $u_{0}$ such that

$$
\sup _{s \geq 0}\left\{\|v(s)\|_{r+1}^{r+1}\right\} \leq K_{0}\left(K_{1}^{r+1}+(1+r)^{\sigma_{0}} \sup _{s \geq 0}\left\{\|v(s)\|_{\left[(r+m)+\sigma_{0}(1-m)\right] / 2}^{\left[(r+m)+\sigma_{0}(1-m)\right]}\right\}\right) \text {. }
$$

We now define the sequence $\left(r_{j}\right)_{j \geq 0}$ by

$$
1+r_{j+1}=2\left(1+r_{j}\right)-(1-m)\left(\sigma_{0}-1\right), \quad j \geq 0, \quad r_{0}:=2-m,
$$

and set

$$
V_{j}:=\sup _{s \geq 0}\left\{\|v(s)\|_{r_{j}+1}^{r_{j}+1}\right\}, \quad j \geq 0
$$


For $j \geq 0$, we take $r=r_{j+1}$ in (2.20) and realize that

$$
\begin{aligned}
V_{j+1} & \leq K_{0}\left(K_{1}^{1+r_{j+1}}+\left(1+r_{j+1}\right)^{\sigma_{0}} V_{j}^{2}\right) \\
& \leq K_{0}\left(1+r_{j+1}\right)^{\sigma_{0}} \max \left\{K_{1}^{1+r_{j+1}}, V_{j}^{2}\right\}, \quad j \geq 0 .
\end{aligned}
$$

Since $\sigma_{0}-1<1 /(1-m)$ thanks to the constraint $\zeta>2 / m$, one has $1+r_{0}-(1-m)\left(\sigma_{0}-1\right)>0$ and we are in a position to apply [22, Lemma A.1], which we recall in Lemma 2.3 below for completeness, to conclude that there is $K_{2}>0$ depending only on $m, \zeta, K_{0}$, and $K_{1}$ such that

$$
V_{j}^{1 /\left(1+r_{j}\right)} \leq K_{2}, \quad j \geq 0 .
$$

Equivalently,

$$
\sup _{s \geq 0}\left\{\|v(s)\|_{1+r_{j}}\right\} \leq K_{2}, \quad j \geq 0
$$

and letting $j \rightarrow \infty$ entails that $\|v(s)\|_{\infty} \leq K_{2}$ for all $s \geq 0$, thereby completing the proof of Lemma 2.2.

The proof of Theorem 1.1 for $r \in(1, \infty]$ is now a straightforward consequence of (2.8) and Lemma 2.2.

Lemma 2.3. Let $a>1, b \geq 0, c \in \mathbb{R}, C_{0} \geq 1, C_{1} \geq 1$, and $p_{0}>0$ be given such that $p_{0}(a-1)+c>0$. We define the sequence $\left(p_{k}\right)_{k \geq 0}$ of positive real numbers by $p_{k+1}=a p_{k}+c$ for $k \geq 0$ and assume that $\left(Q_{k}\right)_{k \geq 0}$ is a sequence of positive real numbers satisfying

$$
Q_{0} \leq C_{1}^{p_{0}}, \quad Q_{k+1} \leq C_{0} p_{k+1}^{b} \max \left\{C_{1}^{p_{k+1}}, Q_{k}^{a}\right\}, k \geq 0 .
$$

Then the sequence $\left(Q_{k}^{1 / p_{k}}\right)_{k \geq 0}$ is bounded.

\section{LOWER BOUND NEAR THE EXTINCTION TIME}

We now turn to the lower bound near the extinction time in $L^{m+1}\left(\mathbb{R}^{N}\right)$.

Proof of Theorem 1.2. For $t \in\left[0, T_{e}\right]$, we define

$$
X(t):=\|u(t)\|_{m+1}^{m+1} \text { and } Y(t):=\int_{\mathbb{R}^{N}} u(t, x)^{m+q} d x .
$$

Let $t \in\left(0, T_{e}\right)$. It follows from (1.1a) that

$$
\frac{1}{m+1} \frac{d X}{d t}(t)+\left\|\nabla u^{m}(t)\right\|_{2}^{2}+Y(t)=0 .
$$

Since

$$
1<\frac{m+q}{m}<\frac{m+1}{m}<2^{*}:=\frac{2 N}{(N-2)_{+}}
$$


by (1.8) we infer from the Gagliardo-Nirenberg inequality that

$$
\begin{aligned}
X(t)^{m /(m+1)} & =\left\|u(t)^{m}\right\|_{(m+1) / m} \leq C\left\|\nabla u^{m}(t)\right\|_{2}^{\theta}\left\|u(t)^{m}\right\|_{(m+q) / m}^{1-\theta} \\
& \leq C Y(t)^{m(1-\theta) /(m+q)}\left\|\nabla u^{m}(t)\right\|_{2}^{\theta},
\end{aligned}
$$

where

$$
\theta:=\frac{2 N m(1-q)}{(m+1)[m(N+2)-q(N-2)]}
$$

Consequently, since $u(t) \not \equiv 0$ as $t \in\left(0, T_{e}\right)$,

$$
\left\|\nabla u^{m}(t)\right\|_{2}^{2} \geq C X(t)^{2 m / \theta(m+1)} Y(t)^{-2 m(1-\theta) / \theta(m+q)},
$$

which gives, together with (3.1),

$$
\frac{d X}{d t}(t)+C X(t)^{2 m / \theta(m+1)} Y(t)^{-2 m(1-\theta) / \theta(m+q)}+(m+1) Y(t) \leq 0 .
$$

Setting

$$
\xi:=1+\frac{2 m(1-\theta)}{\theta(m+q)}>1 \quad \text { and } \quad \gamma:=\frac{2 m}{\theta \xi(m+1)},
$$

it follows from Young's inequality that

$$
\begin{aligned}
X(t)^{\gamma} & =X(t)^{\gamma} Y(t)^{-(\xi-1) / \xi} Y(t)^{(\xi-1) / \xi} \leq \frac{1}{\xi} X(t)^{\xi \gamma} Y(t)^{1-\xi}+\frac{\xi-1}{\xi} Y(t) \\
& \leq X(t)^{2 m / \theta(m+1)} Y(t)^{-2 m(1-\theta) / \theta(m+q)}+Y(t)
\end{aligned}
$$

Combining this inequality with (3.2) leads us to the differential inequality

$$
\frac{d X}{d t}(t)+C X(t)^{\gamma} \leq 0, \quad t \in\left(0, T_{e}\right)
$$

Now,

$$
\begin{aligned}
\gamma & =\frac{2 m}{\theta \xi(m+1)}=\frac{2 m(m+q)}{(2 m+\theta(q-m))(m+1)} \\
& =\frac{2 m(m+q)[m(N+2)-q(N-2)]}{2 m\{(m+1)[m(N+2)-q(N-2)]+N(q-m)(1-q)\}} \\
& =\frac{m(N+2)-q(N-2)}{m(N+2)-q N+2} \in(0,1),
\end{aligned}
$$

and we integrate (3.3) over $\left(t, T_{e}\right)$ to obtain

$$
-X(t)^{1-\gamma}+(1-\gamma) C\left(T_{e}-t\right) \leq 0, \quad t \in\left(0, T_{e}\right) .
$$

Noticing that

$$
(m+1) \alpha-N \beta=\frac{m+1}{1-q}-\frac{N(q-m)}{2(1-q)}=\frac{m(N+2)-q N+2}{2(1-q)}=\frac{1}{1-\gamma},
$$

the lower bound (1.11) readily follows from the previous inequality. 
We end up this section with the derivation of the lower bound for $r \in(m+1, \infty]$ from Theorem 1.1 for $r=1$ and Theorem 1.2 .

Proof of Corollary 1.3. We first note that, owing to (1.8), there holds $2 /(q-m)>N$ and (1.9) entails that $u_{0} \in L^{1}\left(\mathbb{R}^{N}\right)$. Since $u_{0}$ also belongs to $L^{\infty}\left(\mathbb{R}^{N}\right)$, we conclude that $u_{0} \in L^{m+1}\left(\mathbb{R}^{N}\right)$.

Let $r \in(m+1, \infty]$ and $t \in\left(0, T_{e}\right)$. We infer from Theorem 1.1, Theorem 1.2, and Hölder's inequality that

$$
\begin{aligned}
c_{m+1}^{m+1}\left(T_{e}-t\right)^{(m+1) \alpha-N \beta} & \leq\|u(t)\|_{m+1}^{m+1} \leq\|u(t)\|_{r}^{r m /(r-1)}\|u(t)\|_{1}^{(r-1-m) /(r-1)} \\
& \leq C_{1}\left(T_{e}-t\right)^{(\alpha-N \beta)(r-1-m) /(r-1)}\|u(t)\|_{r}^{r m /(r-1)},
\end{aligned}
$$

from which (1.12) readily follows.

\section{EVERYWHERE POSITIVITY}

In this section, we assume that $0<m \leq q<1$ and consider a non-negative initial condition $u_{0} \in B C\left(\mathbb{R}^{N}\right), u_{0} \not \equiv 0$. We denote the corresponding solution to (1.1a)-(1.1b) by $u$ and define its extinction time by (1.2). As in [9], the proof relies on an upper bound for $\partial_{t} u$ which we establish now.

Lemma 4.1. For $t>0$ there holds

$$
\partial_{t} u(t) \leq \frac{u(t)}{(1-m) t} \quad \text { in } \quad \mathbb{R}^{N}
$$

When $q=m$, Lemma 4.1] is a consequence of [4, Theorem 2], the proof relying on an homogeneity argument. Though the operator $-\Delta u^{m}+u^{q}$ is not homogeneous, we may still adapt the proof of [4, Theorem 2] when $q \geq m$.

Proof. Given a non-negative initial condition $u_{0} \in B C\left(\mathbb{R}^{N}\right)$, we denote the corresponding solution to (1.1a)-(1.1b) at time $t \geq 0$ by $S(t) u_{0}$. Recall that, if $u_{0}$ and $v_{0}$ are two non-negative functions in $B C\left(\mathbb{R}^{N}\right)$ satisfying $u_{0} \geq v_{0}$, then the comparison principle entails $S(t) u_{0} \geq S(t) v_{0}$ for all $t \geq 0$.

Step 1. We first claim that, for $\lambda \geq 1$,

$$
S(\lambda t) u_{0} \leq \lambda^{1 /(1-m)} S(t)\left(\lambda^{1 /(m-1)} u_{0}\right), \quad t \geq 0 .
$$

Indeed, setting $u(t):=S(t) u_{0}$ for $t \geq 0$, the function $v$ defined by $v(t):=\lambda^{1 /(m-1)} S(\lambda t) u_{0}$ satisfies

$$
\begin{aligned}
\partial_{t} v(t, x)-\Delta v^{m}(t, x)+v(t, x)^{q}= & \lambda^{m /(m-1)} \partial_{t} u(\lambda t, x)-\lambda^{m /(m-1)} \Delta u^{m}(\lambda t, x) \\
& +\lambda^{q /(m-1)} u(\lambda t, x)^{q} \\
= & \left(\lambda^{q /(m-1)}-\lambda^{m /(m-1)}\right) u(\lambda t, x)^{q} \leq 0 .
\end{aligned}
$$

Since $v(0)=\lambda^{1 /(m-1)} u_{0} \leq u_{0}$, we infer from the comparison principle that (4.1) holds true. 
Step 2. Now, fix $t>0$ and consider $h>0$. Since $\lambda=(1+h / t)>1$ and $m \in(0,1)$, we infer from (4.1) and the comparison principle that

$$
\begin{aligned}
S(t+h) u_{0}-S(t) u_{0} & =S(\lambda t) u_{0}-S(t) u_{0} \\
& \leq \lambda^{1 /(1-m)} S(t)\left(\lambda^{1 /(m-1)} u_{0}\right)-S(t) u_{0} \\
& \leq\left[\left(1+\frac{h}{t}\right)^{1 /(1-m)}-1\right] S(t) u_{0} .
\end{aligned}
$$

Dividing the above inequality by $h$ and passing to the limit as $h \rightarrow 0$ complete the proof.

We now argue as in the proof of [9, Lemma 2.5] to complete the proof of Proposition 1.4.

Proof of Proposition 1.4. Fix $t \in\left(0, T_{e}\right)$ and assume for contradiction that $u\left(t, x_{0}\right)=0$ for some $x_{0} \in \mathbb{R}^{N}$. By (1.1a) and Lemma 4.1, there holds

$$
-\Delta u^{m}(t)+u(t)^{q}+\frac{u(t)}{(1-m) t} \geq 0 \quad \text { in } \quad \mathbb{R}^{N},
$$

so that $u(t)^{m}$ is a supersolution to

$$
-\Delta w+d w=0 \quad \text { in } \quad \mathbb{R}^{N}
$$

with $d(x):=u(t, x)^{q-m}+u(t, x)^{1-m} /((1-m) t)$ for $x \in \mathbb{R}^{N}$. Since $t>0$ and $m \leq q<1$, the function $d$ is non-negative and bounded and we infer from the strong maximum principle [16. Theorem 8.19] that $u(t)^{m} \equiv 0$ in $\mathbb{R}^{N}$, contradicting $t<T_{e}$. Consequently, $u(t)^{m}$ is positive everywhere in $\mathbb{R}^{N}$ and the proof of Proposition 1.4 is complete.

\section{ACKNOWLEDGMENTS}

The first author is supported by the ERC Starting Grant GEOFLUIDS 633152. Part of this work was done while the first author enjoyed the hospitality and support of the Institute de Mathématiques de Toulouse, Toulouse, France.

\section{REFERENCES}

[1] U. G. AbDullaev, Instantaneous shrinking of the support of a solution of a nonlinear degenerate parabolic equation, Math. Notes, 63 (1998), pp. 285-292.

[2] N. D. Alikakos, $L^{p}$ bounds of solutions of reaction-diffusion equations, Comm. Partial Differential Equations, 4 (1979), pp. 827-868.

[3] F. Andreu, J. M. Mazón, F. Simondon, And J. Toledo, Attractor for a degenerate nonlinear diffusion problem with nonlinear boundary condition, J. Dynam. Differential Equations, 10 (1998), pp. $347-377$.

[4] P. BÉnilan And M. G. CRAndall, Regularizing effects of homogeneous evolution equations, in Contributions to analysis and geometry (Baltimore, Md., 1980), Johns Hopkins Univ. Press, Baltimore, Md., 1981, pp. 23-39.

[5] M. BorElli AND M. UGHI, The fast diffusion equation with strong absorption: the instantaneous shrinking phenomenon, Rend. Istit. Mat. Univ. Trieste, 26 (1994), pp. 109-140 (1995).

[6] X.-Y. Chen, H. Matano, and M. Mimura, Finite-point extinction and continuity of interfaces in a nonlinear diffusion equation with strong absorption, J. Reine Angew. Math., 459 (1995), pp. 1-36. 
[7] L. C. Evans And B. F. KNerR, Instantaneous shrinking of the support of nonnegative solutions to certain nonlinear parabolic equations and variational inequalities, Illinois J. Math., 23 (1979), pp. 153-166.

[8] R. Ferreira, V. A. Galaktionov, and J. L. Vázquez, Uniqueness of asymptotic profiles for an extinction problem, Nonlinear Anal., 50 (2002), pp. 495-507.

[9] R. Ferreira And J. L. VÁzQuez, Extinction behaviour for fast diffusion equations with absorption, Nonlinear Anal., 43 (2001), pp. 943-985.

[10] J. M. Foster AND D. E. Pelinovsky, Self-similar solutions for reversing interfaces in the slow diffusion equation with strong absorption, SIAM J. Appl. Dyn. Syst., 15 (2016), pp. 2017-2050.

[11] J. M. Foster, C. P. Please, A. D. Fitt, and G. Richardson, The reversing of interfaces in slow diffusion processes with strong absorption, SIAM J. Appl. Math., 72 (2012), pp. 144-162.

[12] A. Friedman And M. A. Herrero, Extinction properties of semilinear heat equations with strong absorption, J. Math. Anal. Appl., 124 (1987), pp. 530-546.

[13] V. A. Galaktionov, S. I. Shmarev, and J. L. VÁzquez, Regularity of interfaces in diffusion processes under the influence of strong absorption, Arch. Ration. Mech. Anal., 149 (1999), pp. 183-212.

[14] V. A. Galaktionov, S. I. Shmarev, and J. L. VÁzquez, Behaviour of interfaces in a diffusion-absorption equation with critical exponents, Interfaces Free Bound., 2 (2000), pp. 425-448.

[15] V. A. Galaktionov and J. L. VÁzquez, Extinction for a quasilinear heat equation with absorption. I. Technique of intersection comparison, Comm. Partial Differential Equations, 19 (1994), pp. 1075-1106.

[16] D. Gilbarg and N. S. Trudinger, Elliptic partial differential equations of second order, Classics in Mathematics, Springer-Verlag, Berlin, 2001. Reprint of the 1998 edition.

[17] M. A. Herrero and J. J. L. Velázquez, Approaching an extinction point in one-dimensional semilinear heat equations with strong absorption, J. Math. Anal. Appl., 170 (1992), pp. 353-381.

[18] R. G. IAGAR And Ph. LaurenÇOt, Extinction for a singular diffusion equation with strong gradient absorption revisited. In preparation.

[19] A. S. Kalashnikov, The nature of the propagation of perturbations in problems of nonlinear heat conduction with absorption, U.S.S.R. Comp. Math. Math. Phys., 14 (1974), pp. 70-85.

[20] - Some problems of the qualitative theory of second-order nonlinear degenerate parabolic equations, Russian Math. Surveys, 42 (1987), pp. 169-222.

[21] R. KERSNER, Nonlinear heat conduction with absorption: space localization and extinction in finite time, SIAM J. Appl. Math., 43 (1983), pp. 1274-1285.

[22] Ph. Laurençot, Solutions to a Penrose-Fife model of phase-field type, J. Math. Anal. Appl., 185 (1994), pp. 262274.

Instituto de Ciencias Matemáticas (ICMAT), Nicolas Cabrera 13-15, Campus de Cantoblanco, E-28049, MADRID, SPAIN

E-mail address: razvan.iagar@icmat.es

Institute of Mathematics of the Romanian Academy, P.O. Box 1-764, RO-014700, Bucharest, RoMANIA.

Institut de Mathématiques de Toulouse, UMR 5219, Université de Toulouse, CNRS, F-31062 Toulouse Cedex 9, France

E-mail address: laurenco@math.univ-toulouse.fr 